\title{
弯晶的引出效率与同步加速器的束流动力学 稳定性
}

吴木营 ${ }^{(1)}$, 罗诗裕 ${ }^{(1 *}$, 郡明珠 ${ }^{(1)}$, 罗晓华 ${ }^{(2)}$ ，姚勇 ${ }^{(4)}$

(1) 东莞理工大学电子工程学院, 东莞 523808 ;

(2) 重庆大学电气工程学院, 重庆 400044;

(3) 重庆交通大学图书馆, 重庆 400074;

(4) 哈尔滨工业大学深圳研究生院, 深圳 518055

*联系人, E-mail: luoshy@126.com

收稿日期: 2011-12-19; 接受日期: 2012-04-19; 网络出版日期: 2012-07-17

东莞市科技计划项目资助(编号: 200910814032)

摘要近年来, 弯晶的束流控制技术得到了迅速发展. 在加速器物理中, 粒子能量和束流强度一直是 人们追求的目标, 而引出技术和引出效率则是保证束流强度的关键. 在经典力学框架内和偶极近似下, 导出了粒子在弯晶中的一般运动方程. 在准静态近似下, 利用哈密顿原理分析了系统的稳定性; 在相空 间密度均匀分布假设下, 利用相面积概念讨论了弯晶的引出效率. 用 Melnikov 方法描述了系统的混沌行 为及临界性质, 给出了常曲率和变曲率弯晶的退道长度.

关键词同步加速器, 周期弯晶, 引出效率, 退道长度, 稳定性

PACS: 29.20.Lg, 29.27.Bd, 05.45.-a, 11.10.Ef

doi: $10.1360 / 132011-1264$

\section{1 引言}

20 世纪 60 年代, 人们在研究带电粒子同晶体相 互作用的过程中发现了沟道效应; 1976 年, 前苏联科 学家 Tsyganov ${ }^{[11}{ }^{1}{ }^{\prime}$ 子运动方向. 1979 年, DUBNA 联合核子研究所的科 学家们在实验上首次发现了弯晶沟道现象, 并同欧 洲核子研究中心(CERN)和列宁格勒的科学家们一道 对这一现象进行了研究. 1984 年, DUBNA 首次实现 了对能量为 $1 \mathrm{GeV}$ 的质子束偏转 ${ }^{[2]} .1989$ 年, 前苏联
高能物理研究所 IHEP 把这一技术纳入了它的中长期 科学发展规划, 并在 1996 年, 实现了对能量为 70 $\mathrm{GeV}$ 质子束的控制 ${ }^{[3,4]}$, 引出效率达到了 $85 \%$, 而束 流强度也达到 $4 \times 10^{12}$ 粒子; 同年, 欧洲 CERN 也实现 了对能量为 $120 \mathrm{GeV}$ 和 $450 \mathrm{GeV}$ 质子束的偏转 ${ }^{[-7]}$; 而美国费米国家实验室(Fermilab)则实现了更高能量 $(900 \mathrm{GeV})$ 的质子束控制 ${ }^{[8]}$. 欧洲大型粒子加速器 LHC 也试图采用这一引出技术.

近年来，弯晶的束流控制技术得到了迅速发

引用格式: 吴木营, 罗诗裕, 邵明珠, 等. 弯晶的引出效率与同步加速器的束流动力学稳定性. 中国科学: 物理学 力学 天文学, 2012, 42: 835-841 Wu M Y, Luo S Y, Shao M Z, et al. The extraction efficiency of bent crystal and beam dynamic stability for synchrotron (in Chinese). Sci Sin-Phys Mech Astron, 2012, 42: 835-841, doi: 10.1360/132011-1264 
展 ${ }^{[9-11]}$. 事实证明, 利用弯晶控制正的带电粒子是非 常有效的, 只需在束流通道上放上一定长度的常曲 率弯晶或变曲率弯晶, 即可实现对束流的准直、切 割、偏转和引出等. 注意到在加速器技术中, 粒子能 量和束流强度是人们始终追求的目标, 而本文关心 的引出技术和引出效率则是提高束流强度的关键. 弯晶的束流控制很大程度上决定于粒子的退道长度, 退道长度越长元件的性能越好, 引出效率越高. 值得 注意的是弯晶的退道长度与系统的稳定性有关. 本 文就试图对这个问题进行分析. 首先, 在经典力学框 架内和偶极近似下, 把粒子在弯晶中的运动方程化 为具有固定力矩、具有阻尼项和受迫项的广义摆方程. 在相空间密度均匀分布假设下, 利用相平面方法分 析了系统的稳定性和弯晶的引出效率. 用 Melnikov 方法讨论了系统的混沌行为及临界性质, 导出了常 曲率和变曲率弯晶的退道长度.

\section{2 运动方程}

设 $(y, z)$ 平面是相邻晶面之间的中心平面, $z$ 轴沿 着束流方向, $x$ 轴垂直中心平面向上. 假设用机械方 法将晶体做常曲率弯曲, 并注意到粒子在 $z$ 方向运动 是相对论的, 而在 $x$ 方向是非相对论的, 粒子的横向 运动可以用经典方法描述. 在牛顿力学框架内和偶 极近似下, 粒子的横向运动方程可以表示为

$$
m \frac{\mathrm{d}^{2} x}{\mathrm{~d} t^{2}}+\frac{\mathrm{d} V(x)}{\mathrm{d} x}=p v \kappa_{0},
$$

其中 $m=m_{0} \gamma, m_{0}$ 是粒子静止质量, $\gamma$ 是相对论因子; $V(x)$ 是粒子-晶体相互作用势, $p v \kappa_{0}$ 是弯晶离心力, $\kappa_{0}$ 是弯晶曲率, $p$ 是粒子动量, $v$ 是粒子速度, 在超 相对论情况下有 $v \approx c$. 假设用声学方法再将晶体做 如下形式 ${ }^{[12,13]}$

$$
x(z)=a \sin \left(2 \pi z / \lambda_{u}\right)
$$

的周期弯曲, 其中 $a$ 是 “振幅”, $\lambda_{u}$ 是晶体摆动场 “波 长”, 如图 1(a)所示. 如果常曲率弯曲的曲率不大, 粒 子的横向坐标可近似表示为

$$
r(t)=\langle R\rangle+x(t),
$$

其中 $\langle R\rangle$ 是弯晶的平均曲率半径. 考虑到晶格热振 动、电子多重散射等因素的影响, 在弯晶沟道中运动 的带电粒子还将受到阻尼作用. 于是, 方程(1)可以 推广为

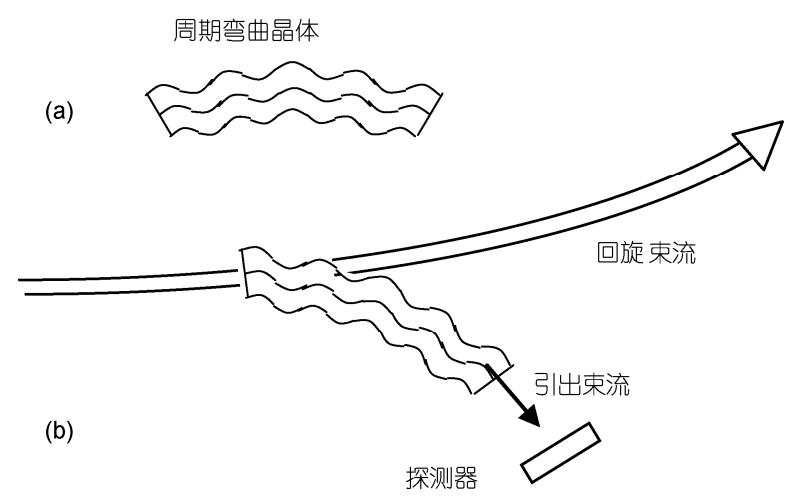

图 1 周期弯晶作为引出元件的示意图

(a) 周期弯曲晶体; (b) 相对配置

Figure 1 The schematic diagram by the periodic bent crystal as extraction cell. (a) The periodic bent crystal; (b) the relative configuration.

$$
m \frac{\mathrm{d}^{2} x}{\mathrm{~d} t^{2}}+2 m \mu_{0} \frac{\mathrm{d} x}{\mathrm{~d} t}+\frac{\mathrm{d}}{\mathrm{d} x} V(x)=p v\left[\kappa_{0}+\kappa(z)\right],
$$

其中 $\mu_{0}$ 是阻尼系数, 物理意义由文献[14]给出, 而

$$
\kappa(z)=\kappa_{m} \sin \frac{2 \pi}{\lambda_{u}} z
$$

是周期弯晶的瞬时曲率，由曲线(2)的二阶导数给出， 其中 $\kappa_{m}=\Omega_{u}^{2} a, \Omega_{u}=2 \pi / \lambda_{u}$ 是晶体摆动场频率, 而 $z \approx c t$.

方程(4)中的相互作用势 $V(x)$ 可以取不同形式 (比如 Lindhard 势、Molierwe 势和正弦平方势等). 引 入正弦平方势 ${ }^{[14-16]}$

$$
V(x)=V_{0} \sin ^{2}(\pi x / d),
$$

其中 $V_{0}$ 是势阱深度, $d$ 是晶面间距, 粒子运动方程 可化为

$$
\frac{\mathrm{d}^{2} \xi}{\mathrm{d} \tau^{2}}+2 \mu \frac{\mathrm{d} \xi}{\mathrm{d} \tau}+\sin \xi=Q+f \cos \omega \tau,
$$

其中

$$
\begin{gathered}
\xi=2 \pi x / d, \quad \delta=\frac{2 \pi^{2} V_{0}}{m d^{2}}, \quad \tau=\delta^{1 / 2} t, \\
\mu=\mu_{0} / \delta^{1 / 2}, \quad \omega=\Omega_{u} / \delta^{1 / 2}, \\
Q=\frac{2 \pi p v \kappa_{0}}{m \delta d}, \quad f=\frac{2 \pi p v \kappa_{m}}{m \delta d} .
\end{gathered}
$$

方程(7)描述了粒子在弯晶中的运动行为. $Q=0$ 描写了粒子在周期弯晶中的运动行为; $f \cos \omega \tau=0$, 描写了粒子在常曲率弯晶中的运动行为. 从实际考 
虑, 可假设方程(7)中阻尼项和受迫项的大小为 $O\left(\varepsilon^{1}\right)$ 量级, 其他项为 $O\left(\varepsilon^{0}\right)$ 量级. 于是, 方程(7)可改写为

$$
\frac{\mathrm{d}^{2} \xi}{\mathrm{d} \tau^{2}}+\sin \xi=Q+\varepsilon\left(-2 \mu \frac{\mathrm{d} \xi}{\mathrm{d} \tau}+f \cos \omega \tau\right),
$$

其中 $\varepsilon$ 是小参数, 表示伴随它的项是小项. 我们关 心的是弯晶引出效率, 而弯晶引出效率与系统的 稳定性和退道长度有关. 首先讨论系统稳定性, 再 讨论粒子的退道长度.

\section{3 稳定性}

\section{1 常曲率弯晶的稳定性}

当 $\varepsilon=0$ 时, 方程(10)化为

$$
\frac{\mathrm{d}^{2} \xi}{\mathrm{d} \tau^{2}}+\sin \xi=Q,
$$

描写了无阻尼情况下, 粒子在常曲率弯晶中的运动 行为. 系统(11)的 Hamiltonian 由

$$
H=\frac{1}{2} \varsigma^{2}-\left[\cos \xi-\cos \xi_{s}+\left(\xi-\xi_{s}\right) \sin \xi_{s}\right]
$$

给出, 其中 $\xi_{s}=\arcsin Q$. 下面用相平面方法对 $Q=0$ 和 $Q \neq 0$ 两种情形进行讨论, 并导出弯晶的引出 效率.

\subsection{1 $Q=0$ 情形}

对于 $Q=0$ 的情形, 方程(11)描述了粒子在直单 晶中的运动行为. 系统 Hamiltonian 为

$$
H_{0}=\frac{1}{2} \varsigma^{2}+2 \sin ^{2} \frac{\xi}{2} \text {. }
$$

根据 $H_{0}$ 的大小, 相平面上的轨道分为三类. 其中, $H_{0}=2$ 的轨道是异宿轨道:

$$
\begin{aligned}
& \xi= \pm 2 \arcsin (s h \tau), \\
& \varsigma= \pm 2 \sec h \tau .
\end{aligned}
$$

$0<H_{0}<2$ 的轨道是周期轨道:

$$
\begin{aligned}
& \xi=2 \arcsin (\kappa s n \tau), \\
& \varsigma=2 \kappa c n \tau,
\end{aligned}
$$

其中 $s n \tau$ 和 $c n \tau$ 是 Jacobian 椭园函数, $\kappa=H_{0} / 2$ 是椭 圆函数的模.

做正则变换

$$
\sin \frac{\xi}{2}=\kappa \sin w, \quad \frac{\varsigma}{2}=\kappa \cos w,
$$

可将 Hamiltonian (13)化为最简单形式

$$
H_{0}=2 \kappa^{2} \text {. }
$$

引入作用量

$$
J=\frac{1}{2 \pi} \oint \varsigma \mathrm{d} \xi
$$

其中 $\xi, \varsigma$ 由式(15)给出. 将式(15)代入式(18)并完成 积分, 可得

$$
J=\frac{1}{2 \pi} \oint \varsigma \mathrm{d} \xi=\frac{8}{\pi}\left[E(\kappa)-(1-\kappa)^{2} K(\kappa)\right] .
$$

$K(\kappa)$ 和 $E(\kappa)$ 是第一类和第二类全椭圆积分. 由式 (19)可以看出, 作用量实际上是束流的相面积在一个 周期内的平均值. 当 $\kappa=1$ 时作用量最大 $J_{\text {max }}=8 / \pi$. 相应的轨道是分支轨道(异宿轨道), 异宿轨道包围的 面积

$$
A_{h}=2 \pi J_{\max }=16 .
$$

\subsection{2 $Q \neq 0$ 情形}

$Q \neq 0$ 的情形描写了粒子在常曲率弯晶中的运 动行为. 分支线(同宿轨道)的 Hamiltonian

$$
H_{s x}=2 \cos \xi_{s}-\left(\pi-2 \xi_{s}\right) \sin \xi_{s} .
$$

这条轨道有两个零点 $\xi_{u}$ 和 $\pi-\xi_{s}$, 其中一个零点 $\xi_{u}$ 由方程

$$
\cos \xi_{u}+\xi_{u} \sin \xi_{s}=-\cos \xi_{s}+\left(\pi-\xi_{s}\right) \sin \xi_{s}
$$

给出. 同宿轨道包围的面积

$$
A_{s}=\oint \varsigma_{s x}(\xi) \mathrm{d} \xi=16 \eta\left(\xi_{s}\right) .
$$

由式(20)和(23)可以看出, $\eta\left(\xi_{s}\right)=A_{s} / A_{h}$ 是 $\xi_{s} \neq 0$ (或 $Q \neq 0$ ) 和 $\xi_{s}=0$ (或 $Q=0$ ) 时分支线包围的面积比

$$
\begin{aligned}
& \eta\left(\xi_{s}\right) \\
& =\frac{1}{4 \sqrt{2}} \int_{\xi_{u}}^{\pi-\xi_{s}}\left[\cos \xi-\cos \xi_{s}-\left(\pi-\xi-\xi_{s}\right)\right]^{1 / 2} \mathrm{~d} \xi,
\end{aligned}
$$

且可近似由公式

$$
\eta\left(\xi_{s}\right) \approx \frac{1-\sin \xi_{s}}{1+\sin \xi_{s}}
$$

给出, 其中 $0 \leqslant \eta\left(\xi_{s} \leqslant 1\right)$. 图 2 给出了 $Q=0,0.2,0.4$, $0.6,0.8,1$ 等情况下的分支轨道, 对应于 $Q=0$ 的分支 轨道是异宿轨道, 相面积由式(20)给出, 如图 2 中的 橄榄形区域所示. $Q \neq 0$ 的分支轨道是同宿轨道, 相 面积由式(23)给出，如图 2 中的 $\alpha$ 形区域所示. 


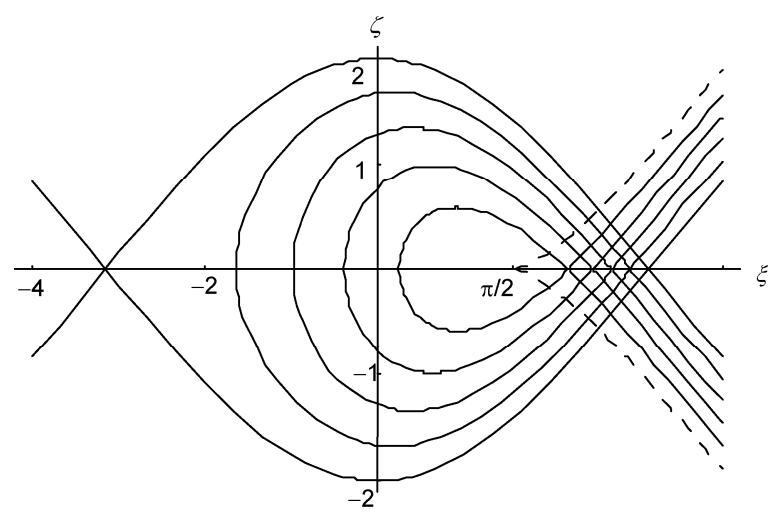

图 2 系统的同宿轨道和异宿轨道

从外到内 $Q=0,0.2,0.4,0.6,0.8,1.0$

Figure 2 The homoclinic orbits and the heteroclinic orbits of the system. From outside to inside: $Q=0,0.2,0.4,0.6,0.8,1.0$.

\subsection{3 引出效率}

在相空间密度均匀分布假设下, $\eta\left(\xi_{s}\right)$ 描述了弯 晶对束流引出的影响, 不妨将这个因子定义为常曲 率弯晶的引出效率. 选择单晶 $\operatorname{Si}(110)$ 做引出元件, 相应的参数 $V_{0}=27 \mathrm{eV}$, 晶面间距 $d=3.84 \times 10^{-10} \mathrm{~m}$, 假设曲率半径为 $1 \mathrm{~m}$, 对于能量为 $70 \mathrm{GeV}$ 的质子, 可 由式(8), (9)和 $\xi_{s}=\arcsin Q$ 算出 $\xi_{s}=8.99^{\circ}$, 再由式(25) 可得弯晶硅的引出效率为 $73.4 \%$. 当初, 前苏联高能 物理研究所用弯晶硅引出 $70 \mathrm{GeV}$ 质子束时引出效率 为 $85 \%$ [3,4].

\section{2 变曲率弯晶的稳定性}

方程 (7) 描写的系统是一个典型的动力学系 统. 下面用 Melnikov 方法对异宿轨道的混沌行为进 行分析.

\subsection{1 异宿轨道的 Melnikov 函数与 Smale 马蹄变 换意义下的混沌行为}

对于无扰动系统(10)的异宿轨道, 可构造如下形 式的 Melnikov 函数:

$$
\begin{aligned}
M_{ \pm}^{s}\left(\tau_{0}\right) & =\int_{-\infty}^{+\infty} \varsigma_{ \pm}(\tau)\left[Q-2 \mu \varsigma_{ \pm}^{s}(\tau)+f \sin \omega\left(\tau+\tau_{0}\right)\right] \mathrm{d} \tau \\
& = \pm \frac{2 f \pi}{\operatorname{ch}\left(\frac{\pi}{2} \omega\right)}\left[2 \mu / \delta_{ \pm}^{s}+\sin \omega \tau_{0}\right],
\end{aligned}
$$

其中 $\varsigma_{ \pm}$由方程(14)第二式给出, 而

$$
\delta_{ \pm}=\frac{c_{0}}{b \mp b_{0}} ; \quad b=Q / 2 \mu ; b_{0}=4 / \pi ; \quad c_{0}=f\left(\operatorname{ch} \frac{\pi}{2} \omega\right)^{-1} .
$$

在参数 $(Q, \mu)$ 平面上, 如果条件

$$
2 \mu<\left|\delta_{ \pm}\right|
$$

满足, 则对于充分小的 $\varepsilon$, 系统(10)的 Poincare 映射 在两个不动点 $[(-\pi, 0),(\pi, 0)]$ 的稳定流形和不稳定流 形将横向交截, 即 Poincare 映射具有 Smale 马蹄变 换意义下的混沌行为.

将式(27)代入式(28), 可将 Smale 马蹄的混沌条 件表示为

$$
\left|\frac{Q \mp 8 \mu / \pi}{f}\right|<\sec h(\pi \omega / 2) .
$$

上式中取正号的条件, 自然满足取负号的情形. 于是, 只需讨论如下的混沌条件即可:

$$
\left|\frac{Q-8 \mu / \pi}{f}\right|<\sec h(\pi \omega / 2) \text {. }
$$

\subsection{2 混沌振荡的物理意义}

将式(8)和(9)代入式(30), 混沌条件可用系统的 原始参数表示为

$$
\left|\frac{\frac{\kappa_{0}}{d}-\frac{4 \mu_{0} \sqrt{2 m V_{0}}}{\pi^{2} p v}}{\kappa_{m}}\right|<\pi \operatorname{sech}\left(d \Omega_{u} \sqrt{m / 8 V_{0}}\right) .
$$

以下为讨论:

(1) 从条件(31)可以看出, 如果系统不存在周期 弯曲, 即 $\kappa_{m}=0$, 不等式左端为无穷, 条件(31)永远 不满足, 系统不存在 Smale 马蹄变换意义下的混沌. 注意到 $\kappa_{m}=0$ 的系统是常曲率系统, 这就是说, 常曲 率系统是稳定系统.

(2) 从式(31)还可以看出, 系统的曲率越大混沌 条件越容易满足. 当它达到

$$
\left(\kappa_{m}\right)_{\mathrm{c}}=\left|\left(\frac{\kappa_{0}}{\pi d}-\frac{4 \mu_{0} \sqrt{2 m V_{0}}}{\pi^{3} p v}\right) \cosh \left(d \Omega_{u} \sqrt{m / 8 V_{0}}\right)\right|
$$

时, 系统处于临界状态. 当 $\kappa_{m}<\left(\kappa_{m}\right)_{\mathrm{c}}$ 时系统是稳定 的; 当 $\kappa_{m}>\left(\kappa_{m}\right)_{\mathrm{c}}$ 时系统不稳定, 而且存在 Smale 马 蹄变换意义上的混沌振荡. 注意到 $\kappa_{m}=\Omega_{u} a, \kappa_{m}$ 越 大周期弯曲的幅值 $a$ 就越大, 而 $a$ 越大系统就可能越 不稳定. 注意到作为束流引出元件的周期弯晶又常 
常用来获得摆动场辐射, 在人们研究摆动场辐射时 发现 $a$ 越大辐射强度越强. 于是, 为了获得较强的摆 动场辐射, 就试图将周期弯晶的振幅做得越大越 好. 而现在的分析表明, 振幅 $a$ 太大系统可能不稳 定. 如何将二者折中考虑是研究晶体摆动场辐射的 新课题.

(3) 当不等式(31)左端分子为零, 即系统处于阻 尼系数

$$
\mu_{0}=\frac{\pi^{2} p v \kappa_{0}}{4 d \sqrt{2 m V_{0}}}
$$

附近时, 条件(31)很容易满足, 任何扰动都可能导致 系统出现混沌振荡. 由式(31) 可得阻尼系数 $\mu_{0}$ 的临 界值为

$$
\left(\mu_{0}\right)_{\mathrm{c}}=\frac{\pi^{2} p v\left(\pi \kappa_{m} \operatorname{sech}\left(d \Omega_{u} \sqrt{m / 8 V_{0}}\right)-\kappa_{0} / d\right)}{4 \sqrt{2 m V_{0}}} .
$$

当阻尼系数 $\mu_{0}>\left(\mu_{0}\right)_{\mathrm{c}}$ 时系统是稳定的, 否则不稳定. 这就是说, 仅当阻尼系数足够大时, 才有可能抑制混 沌的发生.

\section{4 退道长度}

由于晶体弯曲、电子多重散射和晶格热振动等因 素的影响, 粒子的横向能量将不断增加, 当它的横向 能量大于势阱深度时, 粒子将离开沟道, 这个现象称 为退道. 退道现象会使束流强度减弱, 是弯晶束流处 理技术中应当尽量避免和克服的.

\section{1 常曲率弯晶的退道长度}

使用晶体来准直、偏转和引出束流时, 退道长度 这个量十分重要. 注意到当带电粒子同物质相互作 用时, 由于退道效应, 束流强度会越来越小. 设 $n(z)$ 是晶体厚度为 $z$ 处的束流密度, 距离变化 $\mathrm{d} z$ 后束流密 度的减少量 $\mathrm{d} n(z)$ 可表示为

$$
\mathrm{d} n(z)=-\lambda n(z) \mathrm{d} z,
$$

其中 $\lambda$ 是粒子的退道常数, 积分(35)式, 可得

$$
n(z)=n_{0} \mathrm{e}^{-\lambda z},
$$

$n_{0}$ 是 $z=0$ 处的束流密度. 定义束流密度减少为原来 $1 / e$ 时的晶体长度为退道长度, 并用符号 $L_{d}$ 表示. 对于给定的晶体, 退道长度与粒子能量 $\gamma$ 和曲率半 径 $R$ 有关. 考虑到晶格热振动和电子多重散射, 常曲
率弯晶的退道长度 $L_{d}(\gamma, R)$ 可表示为

$$
L_{d}(\gamma, R)=\left(1-\frac{R_{\mathrm{c}}}{R}\right) L_{d}(\gamma, \infty)
$$

其中 $R_{\mathrm{c}}=E / V_{\max }^{\prime}$ 是临界曲率半径, $E=m_{0} c^{2}(\gamma-1)$ 是 粒子能量, $V_{\text {max }}^{\prime}$ 是最大离心力, 而 $L_{d}(\gamma, \infty)$ 是直单晶 的退道长度, 从原子碰撞和电子多重散射理论可 导出

$$
L_{d}(\gamma, \infty)=\frac{256 \gamma}{9 \pi^{2}} \frac{M}{Z} \frac{a_{\mathrm{TF}}}{r_{\mathrm{c}}} \frac{d}{\Lambda},
$$

其中 $a_{\mathrm{TF}}$ 是 Thomas-Fermi 屏蔽距离, $r_{\mathrm{c}}=2.8 \times 10^{-15}$ $\mathrm{m}$ 是电子经典半径, $Z$ 和 $M$ 是粒子的电荷数(以电子 电荷为单位) 和质量数 (以电子质量为单位), 而 $\Lambda$ 是 Coulomb 对数.

\section{2 变曲率弯晶的退道长度}

对于变曲率弯晶, 曲率 $\kappa(z)=\kappa_{m} \sin \left(2 \pi z / \lambda_{u}\right)$ 不 再是常数, 引入平均曲率

$$
\begin{aligned}
\langle\kappa(z)\rangle & =\frac{1}{\lambda_{u}} \int_{0}^{\lambda_{u}} \kappa_{u}^{2} a_{u}\left|\sin \kappa_{u} z\right| \mathrm{d} z \\
& =\frac{2}{\pi R_{\min }}=\frac{2}{\pi} \kappa_{m},
\end{aligned}
$$

退道长度 $L_{d}(\gamma,\langle R\rangle)$ 可表示为

$$
L_{d}(\gamma,\langle R\rangle)=\left(1-\frac{2}{\pi} C\right) \gamma \alpha_{d}(\gamma),
$$

其中 $C=\varepsilon \kappa_{m} / V^{\prime}, \quad \varepsilon$ 是粒子能量, $V$ 是粒子-晶体相 互作用势; $\alpha_{d}(\gamma)$ 是约化退道长度. 由式(38)和(40)可 以看出, 退道长度 $L_{d}$ 与粒子能量 (相对论因子 $\gamma$ ) 成 正比, 能量越高, 退道长度 $L_{d}$ 越长. 对于 $\mathrm{MeV}$ 范围 的粒子, 退道长度只有微米量级; 对于 $\mathrm{TeV}$ 范围的粒 子, 退道长度可达到米以上. 这就决定了弯晶的束流 控制首先在高能范围实现. 对于中低能加速器的弯 晶束流控制正在研究中.

\section{5 结论}

近年来，弯晶的束流控制技术得到了迅速发展. 弯晶的束流控制很大程度上决定于粒子的退道长度, 而粒子退道长度与粒子的能量成正比, 能量越高退 道长度越长. 退道长度问题是一个稳定性问题, 也是 一个引出效率问题. 如何提高引出效率是人们一直 
关心的问题. 考虑到晶体弯曲、晶格热振动、电子多 重散射和相互作用势的非线性等因素的影响，在经 典力学框架内和偶极近似下, 把粒子的运动方程化 为具有常数力矩、阻尼项和受迫项的摆方程. 在相空
间密度均匀分布假设下, 利用相平面方法讨论了系 统的稳定性和弯晶的引出效率. 用 Melnikov 方法讨 论了异宿轨道的混沌行为及系统的临界性质, 导出 了常曲率和变曲率弯晶的退道长度.

\section{参考文献}

1 Tsyganov E N. Some aspect of the mechanism of a charge particle penetration through a monocrystal. FermiLab, 1976, TM-682

2 Andreev V A, Bauublis V V, Damaskinskij E A, et al. Focusing of a beam $1 \mathrm{GeV}$ proton during volume capture in a channeling mode by the monocrystal. Pisma ZhETF, 1984, 39(2): 58-61

3 Asseev A A, Gorin M Y. Crystal-aided non-resonat extraction of $70 \mathrm{GeV}$ proton from the IHEP accelerator. Nucl Instr Meth, 1996, B119: 210-214

4 Chesnokov Y A. Review of IHEP experiments for focusing and deflection 70 GeV proton beam with bent crystal. Nucl Instr Meth, 1996, B119: 163-171

5 Elsener K, Fidecano G, Gir M, et al. Proton extraction from the CERN-SPS using bent silicon crystal. Nucl Instr Meth, 1996, B119: $215-230$

6 Bauricheter A, Kirsebom K, Medeuwadt R, et al. New results from the CERN-SPS beam deflection eaperiments with bent crystal. Nucl Instr Meth, 1996, B119: 172-180

7 Doble N, Gatigton L, Grafstrom P A. Novel application of bent crystal channeling to the production simultaneous particle beams. Nucl Instr Meth, 1996, B119: 181-190

8 Murphy C T, Carrigan R, Chen D, et al. First results from bent crystal extraction at the Fermilab Tevatron. Nucl Instr Meth,1996, B119: 231-238

9 Bryukov V M, Afonin A G, Baranov V T, et al. Accelerator test of crystal undulators. In: Wiedemann H, ed. Advance Radiation Sources and Application. Berlin: Springer, 2006. 191-200

10 Luo S Y, Shao M Z, Hu X D, et al. Average field idea and single particle model for 2-dimension crystallization beams (I) (in Chinese). High Phys Nucl Phys, 2004, 28(1): 96-99 [罗诗裕, 邵明珠，胡西多，等.二维晶化束的平均场概念与单粒子模型(I). 高能物理与核物理， 2004, 28(1): 96-99]

11 Hu X D, Luo S Y, Shao M Z, et al. Average field idea and single particle model for 2-dimension crystallization beams (II) (in Chinese). High Phys Nucl Phys, 2004, 28(2): 196-199 [胡西多，罗诗裕，邵明珠，等.二维晶化束的平均场概念与单粒子模型(II). 高能物理与核 物理, 2004, 28(2): 196-199]

12 Korol A V, Solovyov A V, Greiner W, et al. Photon emission in crystalline undulator. Nucl Instr Methods Phys Res, 2008, B266: 1173-1176

13 Tabrizi M, Korol A V, Solovyov A V, et al. Feasibility of an electron-based crystalline undulator. Phys Rev Lett, 2007, 98: 164801

14 Luo S Y, Li H T, Wu M Y, et al. The resonance behaviour and dynamic stabilities of strained superlattice (in Chinese). Acta Phys Sin, 2010, 59(8): 5766-5771 [罗诗裕, 李洪涛, 吴木营, 等. 应变超晶格系统的共振行为及其动力学稳定性. 物理学报, 2010, 59(8): 5766-5771

15 Luo S Y, Shao M Z, Luo X H. The global bifurcation and chaotic behaviours for the crystalline undulator radiation (in Chinese). Acta Phys Sin，2010，59(4)：2685-2690 [罗诗裕，郡明珠，罗晓华. 晶体摆动场辐射系统的全局分叉与混沌行为. 物理学报，2010，59(4): 2685-2690]

16 Luo S Y, Shao M Z, Luo X H. The sine-squared potential and dislocation dynamics for strained superlattice (in Chinese). Sci Sin-Phys Mech Astron, 2010, 40(2): 207-212 [罗诗裕, 邵明珠, 罗晓华. 正弦平方势与应变超晶格位错动力学. 中国科学: 物理学 力学 天文 学, 2010,40(2): 207-212] 


\title{
The extraction efficiency of bent crystal and beam dynamic stability for synchrotron
}

\author{
WU MuYing ${ }^{1}$, LUO ShiYu ${ }^{1}$, SHAO MingZhu ${ }^{1}$, LUO XiaoHua ${ }^{2,3} \&$ YAO Yong ${ }^{4}$ \\ ${ }^{1}$ College of Electronic Engineering, Dongguan University of Technology, Dongguan 523808 China; \\ ${ }^{2}$ Electric Engineering College of Chongqing University, Chongqing 400044, China; \\ ${ }^{3}$ Library, Chongqing University of Communications, Chongqing 400074, China; \\ ${ }^{4}$ Harbin Institute of Technology Shenzhen Graduate School, Shenzhen 518055, China
}

In recent years, the control technology of beam by bent crystal has been developed rapidly. In accelerator physics, the particle energy and the beam intensity are looked into one goal, and extraction technology and extraction efficiency are the keys to ensure the beam intensity. Within the framework of classical mechanics and in the dipole approximation, the general motion equation of the particles in the bent crystal is derived. In the quasi-static approximation, the stability of the system by using Hamilton's principle is analysed. In the uniform distribution assumption of the phase space density, the extraction efficiency in the bent crystal by the concept of phase area is discussed. We describe the chaotic behavior and the critical properties of the system by Melnikov method and give the dechannelling length of the bent crystal with the constant curvature and variable curvature.

synchrotron, bent crystal, extraction efficiency, dechannelling length, stability

PACS: $29.20 . \mathrm{Lg}, 29.27 . \mathrm{Bd}, 05.45 .-\mathrm{a}, 11.10 . \mathrm{Ef}$

doi: $10.1360 / 132011-1264$ 